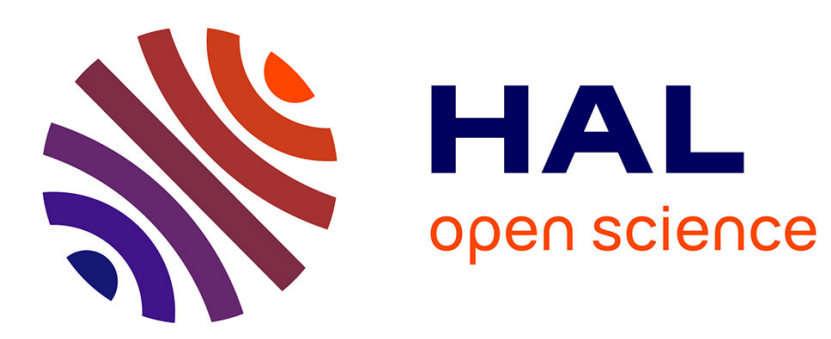

\title{
Asymptotic modeling of thin linearly quasicrystalline plates
}

\author{
Thibaut Weller, Christian Licht
}

\section{To cite this version:}

Thibaut Weller, Christian Licht. Asymptotic modeling of thin linearly quasicrystalline plates. Comptes Rendus Mécanique, 2013, 341, pp.793-798. 10.1016/j.crme.2013.10.002 • hal-01232600

\section{HAL Id: hal-01232600 https://hal.science/hal-01232600}

Submitted on 23 Nov 2015

HAL is a multi-disciplinary open access archive for the deposit and dissemination of scientific research documents, whether they are published or not. The documents may come from teaching and research institutions in France or abroad, or from public or private research centers.
L'archive ouverte pluridisciplinaire HAL, est destinée au dépôt et à la diffusion de documents scientifiques de niveau recherche, publiés ou non, émanant des établissements d'enseignement et de recherche français ou étrangers, des laboratoires publics ou privés. 


\title{
Asymptotic modeling of thin linearly quasicrystalline plates
}

\section{Analyse asymptotique de plaques minces quasicristallines linéaires}

\author{
Thibaut Weller*, Christian Licht \\ Laboratoire de mécanique et génie civil, UMR 5508 CNRS - UMII, Université Montpellier-2, c.c. 048, place Eugène-Bataillon, 34095 Montpellier \\ cedex 5, France
}

\begin{abstract}
A B S T R A C T
We rigorously derive a theory of thin linearly quasicrystalline plates by studying the limit behavior of a three-dimensional flat body as its thickness tends to zero. We exhibit the existence of 26 different models, each of them linked to a specific set of boundary conditions. This stunning number of models is essentially the consequence of the coupling between displacements and a specific local rearrangement of matter at the microscopic scale that is called a phason. We exhibit the influence of the icosahedral order on the limit behavior.
\end{abstract}

\section{R É S U M É}

L'analyse asymptotique, lorsque l'épaisseur tend vers 0 , de plaques minces quasicristallines linéaires montre que, selon le type de conditions aux limites considéré, il apparaît 26 modèles rendant compte de comportements différents. Ce nombre étonnamment élevé de modèles limites est essentiellement dû au couplage entre les déplacements élastiques et un type spécifique de réarrangement atomique appelé phason. On montre en particulier l'influence de l'ordre icosahédral sur ces différents comportements limites.

\section{Introduction}

As a new phase of solid matter, quasicrystals - henceforth abbreviated as QCs - were first discovered and studied by D. Shechtman. At the time of their discovery, in 1982, they presented a fundamental challenge to theoretical physicists to re-examine traditional concepts and devise new analytical methods for determining their physical properties. Indeed, it had been generally assumed that pentagonal symmetry is impossible in solids, that Bragg diffraction peaks imply periodic translational order, that all pure solids are either crystalline or glassy, and that the (classically) stable state of a solid is crystalline. The discovery of icosahedral phases in metallic alloys by Shechtman et al. [1] has compelled theorists to reconsider these assumptions and to confront many new problems (see [2] for a comprehensive introduction to the subject). Therefore, the physical properties such as the structural, electronic, magnetic, optical and thermal characteristics of QCs have been investigated intensively. Most of these properties combine effectively to give interesting technological applications which have been protected recently by several patents [3-5]. As an example among others, the combination of such kind of properties as high hardness, low friction and corrosive resistance with bio-compatibility is very promising for introducing QCs in surgical applications. It is thus of major technological interest to provide efficient modelings of quasicrystalline

\footnotetext{
* Corresponding author.

E-mail addresses: thibaut.weller@univ-montp2.fr (T. Weller), clicht@univ-montp2.fr (C. Licht).
} 
structures. Here we perform a mathematical modeling of linear quasicrystalline plates by regarding the thickness as a small parameter denoted by $\varepsilon$. Then we study the behavior of the solution of the physical problem as $\varepsilon$ tends to 0 . We show that depending on the type of boundary conditions, 26 different models indexed by a triplet $p=\left(p_{1}, p_{2}, p_{3}\right) \in\{0,1,2\}^{3}$ appear at the limit. Comparing to our previous studies devoted to the mathematical modeling of thin plates in the framework of multi-physical couplings [6-8], we are here in front of a real 'explosion' of the number of limit models. This multiplication of models, however, has its roots in the very structure of QCs. As shown in [10], the constitutive law of quasicrystalline media present a coupling between two different kinds of displacement fields: the phonon field, denoted by $u$, and the phason field, denoted by $w$ (see (2)). Roughly speaking, the existence of the phason field $w$ is due to the fact that the dimension of the reciprocal space of a three-dimensional QC is greater than three. Whereas phonons are related to translation of atoms and therefore to classical elastic displacements, phasons are associated with atomic rearrangements and appear in the constitutive equations only through their gradients and not their symmetrized gradients. As it will be explained infra, this is the main cause of the huge amount of models we obtain for a single quasicrystalline thin plate. For all the models we get, we are able to express the limit constitutive law as a Schur complement of the tensor accounting for the coupling between phonon and phason fields. Finally, we study the influence of the icosahedral order on all our models and show that a decoupling between phasons and phonons may appear in the structure.

\section{Setting the problem}

We will denote phonon fields by the letters $u, v$ and $v^{\prime}$ while the phason fields will be denoted by $w, \psi$ and $\psi^{\prime}$. Depending on the nature of our formulation, these letters and some other symbols may be indexed by $\varepsilon$ which stands for the thickness of the plate, regarded as a parameter. Classically, the linearized strain tensor is written $e(u) \in \mathbb{S}^{3}$ where $\mathbb{S}^{N}$ indicates the set of all $N \times N$ real and symmetric matrices. Used as indices, letters $i$ and $j$ take their values in $\{1,2,3\}$ while $\alpha$ and $\beta$ take their values in $\{1,2\}$. When there is no ambiguity, the convention of summation over repeated indices is understood. We recall that $2 e_{i j}(u)=\partial_{i} u_{j}+\partial_{j} u_{i}$ where the symbol $\partial_{i}$ refers to the partial derivative with respect to the $i$-th coordinate. The gradient of a phason field $w$ will be denoted by $\nabla w \in \mathbb{M}^{3}$ where $\mathbb{M}^{N}$ is the set of all $N \times N$ real matrices. The components of $\nabla w$ are given by $(\nabla w)_{i j}=\partial_{j} w_{i}$. Given a physical state $(u, w)$ living in a quasicrystalline medium, we therefore have $(e(u), \nabla w) \in \mathbb{H}$, with:

$$
\mathbb{H}=\mathbb{S}^{3} \times \mathbb{M}^{3}
$$

In the sequel, any element of $\mathbb{H}$ will be denoted by $\mathrm{h}=(\mathrm{e}, \mathrm{g})$. For the sake of simplicity, the classical symbol $\cdot$ will stand for the inner product in $\mathbb{H}, \mathbb{S}^{3}, \mathbb{M}^{3}$ and $\mathbb{R}^{3}$. The set of all linear mappings from a space $X$ into a space $Y$ is denoted by $\mathcal{L}(X, Y)$ and if $X=Y$ we simply write $\mathcal{L}(X)$. In the sequel, for all domain $D$ of $\mathbb{R}^{N}, H_{\Gamma}^{1}(D)$ refers to the subset of the Sobolev space $H^{1}(D)$ whose elements vanish on a part $\Gamma$ of the boundary $\partial D$ of $D$.

The reference configuration of a linearly quasicrystalline thin plate is the closure in $\mathbb{R}^{3}$ of the set $\Omega^{\varepsilon}=\omega \times(-\varepsilon, \varepsilon)$ whose outward unit normal is $n^{\varepsilon}$. Here $\varepsilon$ is a small positive number and $\omega$ is a bounded domain of $\mathbb{R}^{2}$ with a Lipschitz boundary $\partial \omega$. The lateral part of the plate $\partial \omega \times(-\varepsilon, \varepsilon)$ is denoted $\Gamma_{\text {lat }}^{\varepsilon}$, while $\Gamma_{ \pm}^{\varepsilon}=\omega \times\{ \pm \varepsilon\}$ refers to the upper or lower face, respectively. Let $\left(\Gamma_{u D}^{\varepsilon}, \Gamma_{u N}^{\varepsilon}\right),\left(\Gamma_{w D, i}^{\varepsilon}, \Gamma_{w N, i}^{\varepsilon}\right)_{i=1,2,3}$ four suitable partitions of $\partial \Omega^{\varepsilon}$ with $\Gamma_{u D}^{\varepsilon}$ and $\Gamma_{w D, i}^{\varepsilon}$ of strictly positive surface measures. The plate is clamped along $\Gamma_{u D}^{\varepsilon}$ and the phason field $w^{\varepsilon}$ satisfies $w_{i}^{\varepsilon}=w_{0_{i}}^{\varepsilon}$ on $\Gamma_{w D, i}^{\varepsilon}$, where $w_{0}^{\varepsilon}=\left(w_{0_{1}}^{\varepsilon}, w_{0_{2}}^{\varepsilon}, w_{0_{3}}^{\varepsilon}\right)$ is a smooth enough given field defined in $\Omega^{\varepsilon}$. Moreover, the plate is subjected to body forces $f^{\varepsilon}$ and generalized body forces $g^{\varepsilon}$, but also to surface forces $F^{\varepsilon}$ and generalized tractions $G^{\varepsilon}$ of components $G_{i}^{\varepsilon}$ on $\Gamma_{u N}^{\varepsilon}$ and $\Gamma_{w N, i}^{\varepsilon}$, respectively. Finally, we assume that $\Gamma_{u D}^{\varepsilon}=\gamma_{0} \times(-\varepsilon, \varepsilon)$, with $\gamma_{0} \subset \partial \omega$. The equations determining the physical state $s^{\varepsilon}=\left(u^{\varepsilon}, w^{\varepsilon}\right)$ at equilibrium are (see $[10,11]$ for example):

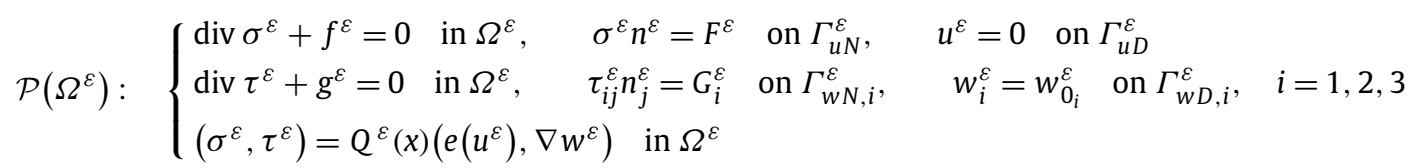

where $u^{\varepsilon}, w^{\varepsilon}, \sigma^{\varepsilon}$ and $\tau^{\varepsilon}$ respectively stand for the phonon field, the phason field, the phonon stress tensor and the phason stress tensor. The operator $Q^{\varepsilon}$ is an element of $\mathcal{L}(\mathbb{H})$ such that:

$$
\left\{\begin{array}{l}
\sigma_{i j}^{\varepsilon}=C_{i j k l}^{\varepsilon} e_{k l}\left(u^{\varepsilon}\right)+R_{i j k l}^{\varepsilon}\left(\nabla w^{\varepsilon}\right)_{k l} \\
\tau_{i j}^{\varepsilon}=R_{k l i j}^{\varepsilon} e_{k l}\left(u^{\varepsilon}\right)+K_{i j k l}^{\varepsilon}\left(\nabla w^{\varepsilon}\right)_{k l}
\end{array}\right.
$$

In these constitutive equations, $C_{i j k l}^{\varepsilon}, K_{i j k l}^{\varepsilon}$ and $R_{i j k l}^{\varepsilon}$ stand for the phonon, phason and phonon-phason coupling coefficients, respectively. As an example, for icosahedral quasicrystals, the stored energy function takes the form (see [12]):

$$
\begin{aligned}
Q^{\varepsilon}\left(e\left(u^{\varepsilon}\right), \nabla w^{\varepsilon}\right) \cdot\left(e\left(u^{\varepsilon}\right), \nabla w^{\varepsilon}\right)= & \frac{1}{2} \lambda^{\varepsilon} e_{i i}\left(u^{\varepsilon}\right) e_{i i}\left(u^{\varepsilon}\right)+\mu^{\varepsilon} e_{i j}\left(u^{\varepsilon}\right) e_{i j}\left(u^{\varepsilon}\right)+\frac{1}{2} K_{1}^{\varepsilon} \partial_{i} w_{j}^{\varepsilon} \partial_{i} w_{j}^{\varepsilon} \\
& +\frac{1}{2} K_{2}^{\varepsilon}\left[\left(\partial_{3}^{\varepsilon} w_{3}^{\varepsilon}\right)^{2}-\left(\partial_{1} w_{3}^{\varepsilon}\right)^{2}-\left(\partial_{2} w_{1}^{\varepsilon}\right)^{2}-\left(\partial_{3} w_{1}^{\varepsilon}\right)^{2}-\left(\partial_{3} w_{2}^{\varepsilon}\right)^{2}\right.
\end{aligned}
$$




$$
\begin{aligned}
& +2\left(\partial_{1} w_{3}^{\varepsilon}+\partial_{3} w_{1}^{\varepsilon}\right) \partial_{1} w_{1}^{\varepsilon}-2\left(\partial_{1} w_{3}^{\varepsilon}-\partial_{3} w_{1}^{\varepsilon}\right) \partial_{2} w_{2}^{\varepsilon} \\
& \left.-2\left(\partial_{2} w_{3}^{\varepsilon}+\partial_{3} w_{2}^{\varepsilon}\right) \partial_{1} w_{2}^{\varepsilon}-2\left(\partial_{2} w_{3}^{\varepsilon}-\partial_{3} w_{2}^{\varepsilon}\right) \partial_{2} w_{1}^{\varepsilon}\right] \\
& +r^{\varepsilon}\left[\left(e_{11}\left(u^{\varepsilon}\right)+e_{22}\left(u^{\varepsilon}\right)-2 e_{33}\left(u^{\varepsilon}\right)\right) \partial_{3} w_{3}^{\varepsilon}+\left(e_{11}\left(u^{\varepsilon}\right)\right.\right. \\
& \left.-e_{22}\left(u^{\varepsilon}\right)\right)\left(\partial_{1} w_{1}^{\varepsilon}+\partial_{2} w_{2}^{\varepsilon}+\partial_{3} w_{1}^{\varepsilon}\right)+2 e_{12}\left(u^{\varepsilon}\right)\left(\partial_{1} w_{2}^{\varepsilon}-\partial_{2} w_{1}^{\varepsilon}-\partial_{3} w_{2}^{\varepsilon}\right) \\
& \left.+2 e_{13}\left(u^{\varepsilon}\right)\left(\partial_{1} w_{1}^{\varepsilon}+\partial_{1} w_{2}^{\varepsilon}-\partial_{2} w_{2}^{\varepsilon}\right)+2 e_{23}\left(u^{\varepsilon}\right)\left(\partial_{2} w_{3}^{\varepsilon}-\partial_{1} w_{2}^{\varepsilon}-\partial_{2} w_{1}^{\varepsilon}\right)\right]
\end{aligned}
$$

where $\lambda^{\varepsilon}, \mu^{\varepsilon}, r^{\varepsilon}, K_{1}^{\varepsilon}$ and $K_{2}^{\varepsilon}$ are five physical coefficients which may depend on $x$ and see [10] for the corresponding form of the tensors $C^{\varepsilon}, R^{\varepsilon}$ and $K^{\varepsilon}$.

We are looking for the state $\left(u^{\varepsilon}, w^{\varepsilon}\right)$ living in the quasicrystalline plate at equilibrium. For this purpose, we first make the following regularity hypothesis on the exterior loading:

$$
\left(\mathbf{H}_{1}\right):\left\{\begin{array}{l}
\left(f^{\varepsilon}, g^{\varepsilon}, F^{\varepsilon}, G_{i}^{\varepsilon}\right) \in L^{2}\left(\Omega^{\varepsilon}\right)^{3} \times L^{2}\left(\Omega^{\varepsilon}\right)^{3} \times L^{2}\left(\Gamma_{u N}^{\varepsilon}\right)^{3} \times L^{2}\left(\Gamma_{w N, i}^{\varepsilon}\right), \quad i=1,2,3 \\
w_{0}^{\varepsilon} \in H^{1}\left(\Omega^{\varepsilon}\right)
\end{array}\right.
$$

Now, on the space of physical states:

$$
V^{\varepsilon}=H_{\Gamma_{u D}^{\varepsilon}}^{1}\left(\Omega^{\varepsilon}\right)^{3} \times H_{\Gamma_{w D, 1}^{\varepsilon}}^{1}\left(\Omega^{\varepsilon}\right) \times H_{\Gamma_{w D, 2}^{\varepsilon}}^{1}\left(\Omega^{\varepsilon}\right) \times H_{\Gamma_{w D, 3}^{\varepsilon}}^{1}\left(\Omega^{\varepsilon}\right)
$$

we define a bilinear form $m^{\varepsilon}$ :

$$
m^{\varepsilon}(\xi, \zeta)=m^{\varepsilon}\left((v, \psi),\left(v^{\prime}, \psi^{\prime}\right)\right)=\int_{\Omega^{\varepsilon}} Q^{\varepsilon}(e(v), \nabla \psi) \cdot\left(e\left(v^{\prime}\right), \nabla \psi^{\prime}\right) \mathrm{d} x^{\varepsilon}
$$

and a linear form $L^{\varepsilon}$ :

$$
L^{\varepsilon}(\xi)=L^{\varepsilon}((v, \psi))=\int_{\Omega^{\varepsilon}}\left(f^{\varepsilon} \cdot v+g^{\varepsilon} \cdot \psi\right) \mathrm{d} x^{\varepsilon}+\int_{\Gamma_{u N}^{\varepsilon}} F^{\varepsilon} \cdot v \mathrm{~d} s^{\varepsilon}+\sum_{i=1}^{3} \int_{\Gamma_{w N, i}^{\varepsilon}} G_{i}^{\varepsilon} \psi_{i} \mathrm{~d} s^{\varepsilon}
$$

The coupled physical problem then takes the form

$$
\mathcal{P}\left(\Omega^{\varepsilon}\right): \quad \text { Find } s^{\varepsilon}=\left(u^{\varepsilon}, w^{\varepsilon}\right) \in\left(0, w_{0}^{\varepsilon}\right)+V^{\varepsilon} \quad \text { such that } m^{\varepsilon}\left(s^{\varepsilon}, \xi\right)=L^{\varepsilon}(\xi), \quad \forall \xi \in V^{\varepsilon}
$$

Thus, with the additional and realistic assumptions of boundedness of $C^{\varepsilon}, R^{\varepsilon}$ and $K^{\varepsilon}$ and of uniform ellipticity of $C^{\varepsilon}$ and $K^{\varepsilon}$ :

$$
\left(\mathbf{H}_{2}\right): \quad Q^{\varepsilon} \in L^{\infty}\left(\Omega^{\varepsilon}, \mathcal{L}(\mathbb{H})\right), \quad \exists \kappa^{\varepsilon}>0 ; \quad Q^{\varepsilon}\left(x^{\varepsilon}\right) \mathrm{h} \cdot \mathrm{h} \geqslant \kappa^{\varepsilon}|\mathrm{h}|_{\mathbb{H}}^{2}, \quad \forall \mathrm{h} \in \mathbb{H}, \quad \text { a.e. } x^{\varepsilon} \in \Omega^{\varepsilon}
$$

the Stampacchia theorem (cf. [13]) implies the

Theorem 2.1. Under assumptions $\left(\mathbf{H}_{1}\right)-\left(\mathbf{H}_{2}\right)$, the problem $\mathcal{P}\left(\Omega^{\varepsilon}\right)$ has a unique solution.

To derive simplified and accurate models, the true question is to study the behavior of $s^{\varepsilon}$ when $\varepsilon$, regarded as a parameter, tends to zero.

\section{The different models}

We will show that 26 different limit behaviors appear according to both the type and magnitude of boundary conditions in $\mathcal{P}\left(\Omega^{\varepsilon}\right)$. These limit behaviors will be indexed by a triplet $p=\left(p_{1}, p_{2}, p_{3}\right)$ of $\{1,2,3\}^{3} \backslash\{0,0,0\}$.

Classically (see [14]) we come down to a fixed open set $\Omega=\omega \times(-1,1)$ through the mapping $\pi^{\varepsilon}$ :

$$
x=\left(x_{1}, x_{2}, x_{3}\right) \in \bar{\Omega} \mapsto \pi^{\varepsilon} x=\left(x_{1}, x_{2}, \varepsilon x_{3}\right) \in \bar{\Omega}^{\varepsilon}
$$

Also, we drop the index $\varepsilon$ for the images by $\left(\pi^{\varepsilon}\right)^{-1}$ of the geometric sets defined in the preceding section. To get physically meaningful results, we have to make various kinds of assumptions. They deal with

(i) the electromechanical coefficients:

$$
\left(\mathbf{H}_{3}\right): \quad Q^{\varepsilon}\left(\pi^{\varepsilon} x\right)=Q(x) \quad \text { with } Q \in L^{\infty}(\Omega, \mathcal{L}(\mathbb{H})), \exists \kappa>0 ; \quad Q(x) \mathrm{h} \cdot \mathrm{h} \geqslant \kappa|\mathrm{h}|_{\mathbb{H}}^{2}, \quad \forall \mathrm{h} \in \mathbb{H}, \quad \text { a.e. } x \in \Omega
$$


(ii) the electromechanical loading:

$$
\left(\mathbf{H}_{4}\right):\left\{\begin{array}{l}
\exists\left(f, g, F, G_{i}\right) \in L^{2}(\Omega)^{3} \times L^{2}(\Omega)^{3} \times L^{2}\left(\Gamma_{u N}\right)^{3} \times L^{2}\left(\Gamma_{w N}^{i}\right), \quad i=1,2,3 \\
f_{\alpha}^{\varepsilon}\left(\pi^{\varepsilon} x\right)=\varepsilon f_{\alpha}(x), \quad f_{3}^{\varepsilon}\left(\pi^{\varepsilon} x\right)=\varepsilon^{2} f_{3}(x), \quad \forall x \in \Omega \\
F_{\alpha}^{\varepsilon}\left(\pi^{\varepsilon} x\right)=\varepsilon^{2} F_{\alpha}(x), \quad F_{3}^{\varepsilon}\left(\pi^{\varepsilon} x\right)=\varepsilon^{3} F_{3}(x), \quad \forall x \in \Gamma_{u N} \cap \Gamma_{ \pm} \\
F_{\alpha}^{\varepsilon}\left(\pi^{\varepsilon} x\right)=\varepsilon F_{\alpha}(x), \quad F_{3}^{\varepsilon}\left(\pi^{\varepsilon} x\right)=\varepsilon^{2} F_{3}(x), \quad \forall x \in \Gamma_{u N} \cap \Gamma_{\text {lat }} \\
g_{i}^{\varepsilon}\left(\pi^{\varepsilon} x\right)=\varepsilon^{2-p_{i}} g_{i}(x), \quad \forall x \in \Omega \\
G_{i}^{\varepsilon}\left(\pi^{\varepsilon} x\right)=\varepsilon^{3-p_{i}} G_{i}(x), \quad \forall x \in \Gamma_{w N, i} \cap \Gamma_{ \pm} \\
G_{i}^{\varepsilon}\left(\pi^{\varepsilon} x\right)=\varepsilon^{2-p_{i}} G_{i}(x), \quad \forall x \in \Gamma_{w N, i} \cap \Gamma_{l a t} \\
w_{0_{i}}^{\varepsilon}\left(\pi^{\varepsilon} x\right)=\varepsilon^{p_{i}} w_{0_{i}}(x), \quad \forall x \in \Omega
\end{array}\right.
$$

(iii) the boundedness of the "work of the exterior loading":

$$
\left(\mathbf{H}_{5}\right): \begin{cases}p_{i}=0: & w_{0_{i}}=0 \\ p_{i}=1: & w_{0_{i}} \text { does not depend on } x_{3} \\ p_{i}=2: & - \text { the closure } \bar{\delta} \text { of the projection of } \Gamma_{w D, i} \text { on } \omega \text { coincides with } \bar{\omega} \\ & - \text { either } G_{i}=0 \text { on } \Gamma_{w N, i} \cap \Gamma_{\text {lat }} \text { or } \Gamma_{w N, i} \cap \Gamma_{\text {lat }}=\emptyset\end{cases}
$$

Also, with the true physical state $s^{\varepsilon}=\left(u^{\varepsilon}, w^{\varepsilon}\right)$ defined on $\Omega^{\varepsilon}$, we associate a scaled physical state $s_{p}(\varepsilon)=(u(\varepsilon)$, w $p(\varepsilon))$ defined on $\Omega$ by:

$$
u_{\alpha}^{\varepsilon}\left(x^{\varepsilon}\right)=\varepsilon u(\varepsilon)_{\alpha}(x), \quad u_{3}^{\varepsilon}\left(x^{\varepsilon}\right)=u(\varepsilon)_{3}(x), \quad w_{i}^{\varepsilon}\left(x^{\varepsilon}\right)=\varepsilon^{p_{i}} w_{p}(\varepsilon)_{i}(x), \quad \forall x^{\varepsilon}=\pi^{\varepsilon} x \in \bar{\Omega}^{\varepsilon}
$$

so that $s_{p}(\varepsilon)$ is the unique solution of the following mathematical problem:

$$
\mathcal{P}(\varepsilon, \Omega)_{p}: \quad\left\{\begin{array}{l}
\text { Find } s_{p}(\varepsilon) \in\left(0, w_{0}\right)+V \quad \text { such that: } \\
m_{p}(\varepsilon)\left(s_{p}(\varepsilon), \xi\right)=L(\xi), \quad \forall \xi \in V=H_{\Gamma_{u D}}^{1}(\Omega)^{3} \times H_{\Gamma_{w D, 1}}^{1}(\Omega) \times H_{\Gamma_{w D, 2}}^{1}(\Omega) \times H_{\Gamma_{w D, 3}}^{1}(\Omega)
\end{array}\right.
$$

equivalent to the genuine physical one, where:

$$
\left\{\begin{array}{l}
m_{p}(\varepsilon)(s, \xi)=\int_{\Omega} Q(x) k_{p}(\varepsilon, s) \cdot k_{p}(\varepsilon, \xi) \mathrm{d} x \\
k_{p}(\varepsilon, \xi)=k_{p}(\varepsilon,(v, \psi))=\left(e(\varepsilon, v), \nabla_{p}(\varepsilon, \psi)\right) \\
e_{\alpha \beta}(\varepsilon, v)=e_{\alpha \beta}(v), \quad e_{\alpha 3}(\varepsilon, v)=\varepsilon^{-1} e_{\alpha 3}(v), \quad e_{33}(\varepsilon, v)=\varepsilon^{-2} e_{33}(v) \\
\nabla_{p}(\varepsilon, \psi)_{i \alpha}=\varepsilon^{p_{i}-1} \partial_{\alpha} \psi_{i}, \quad \nabla_{p}(\varepsilon, \psi)_{i 3}=\varepsilon^{p_{i}-2} \partial_{3} \psi_{i} \\
L(\xi)=L(v, \psi)=\int_{\Omega}(f \cdot v+g \cdot \psi) \mathrm{d} x+\int_{\Gamma_{u N}} F \cdot v \mathrm{~d} s+\sum_{i=1}^{3} \int_{\Gamma_{w N, i}} G_{i} \psi_{i} \mathrm{~d} s
\end{array}\right.
$$

As explained in our previous papers [6-9], the signs of the various powers of $\varepsilon$ in the components of $k_{p}(\varepsilon, \xi)$ induce an orthogonal decomposition of $\mathbb{H}$ in subspaces $\mathbb{H}_{p}^{\star}$ with $\star \in\{-, 0,+\}$, which allows a comprehensive description of our plate models in any theoretically admissible quasicrystallographic classes. We denote by $h_{p}^{\star}$ the projection on $\mathbb{H}_{p}^{\star}$ of any element $\mathrm{h}$ of $\mathbb{H}$. As an example, for $p=(0,1,2)$, we have:

$$
\begin{aligned}
& \mathbb{H}_{p}^{-}=\left\{(\mathrm{e}, \mathrm{g}) \in \mathbb{H} ; \mathrm{e}_{\alpha \beta}=0, \mathrm{~g}_{2 \alpha}=0, \mathrm{~g}_{3 i}=0\right\} \\
& \mathbb{H}_{p}^{0}=\left\{(\mathrm{e}, \mathrm{g}) \in \mathbb{H} ; \mathrm{e}_{i 3}=0, \mathrm{~g}_{1 i}=0, \mathrm{~g}_{23}=0, \mathrm{~g}_{3 \alpha}=0\right\} \\
& \mathbb{H}_{p}^{+}=\left\{(\mathrm{e}, \mathrm{g}) \in \mathbb{H} ; \mathrm{e}_{i j}=0, \mathrm{~g}_{1 i}=0, \mathrm{~g}_{2 i}=0, \mathrm{~g}_{33}=0\right\}
\end{aligned}
$$

Then, for a given triplet $p$, the operator $Q$ can be decomposed in nine elements $Q_{p}^{\star \diamond} \in \mathcal{L}\left(\mathbb{H}_{p}^{\diamond}, \mathbb{H}_{p}^{\star}\right)$ with $\star, \diamond \in\{-, 0,+\}$. Hypothesis $\left(\mathbf{H}_{3}\right)$ on the coefficients implies that $Q_{p}^{00}$ and $Q_{p}^{--}$are positive operators on $\mathbb{H}_{p}^{0}$ and $\mathbb{H}_{p}^{-}$, respectively. Therefore, the Schur complement:

$$
\widetilde{Q}_{p}=Q_{p}^{00}-Q_{p}^{0-}\left(Q_{p}^{--}\right)^{-1} Q_{p}^{-0}
$$

is an element of $\mathcal{L}\left(\mathbb{H}_{p}^{0}\right)$. Note that neither $Q_{p}^{00}$ nor $\widetilde{Q}_{p}$ are necessarily symmetric, but nevertheless:

$$
\kappa\left|\mathrm{h}_{p}^{0}\right|_{\mathbb{H}}^{2} \leqslant \widetilde{Q}_{p}(x) \mathrm{h}_{p}^{0} \cdot \mathrm{h}_{p}^{0}, \quad \forall \mathrm{h}_{p}^{0} \in \mathbb{H}_{p}^{0}, \quad \text { a.e. } x \in \Omega
$$


This is implied by the coercivity of $Q$ (see $\left(\mathbf{H}_{3}\right)$ ) and by the fundamental relation:

$$
\left(Q h_{p}^{-}=h_{p}^{+}=0 \Rightarrow \widetilde{Q}_{p} h_{p}^{0}=(Q h)_{p}^{0} \quad \text { and } \quad \widetilde{Q}_{p} h_{p}^{0} \cdot h_{p}^{0}=Q h \cdot h\right.
$$

The key point of the asymptotic study is to show that if $\bar{k}_{p}$ is the limit (in a suitable topology) of $k_{p}\left(\varepsilon, s_{p}(\varepsilon)\right.$ ), then $\left(Q \bar{k}_{p}\right)_{p}^{-}=\left(\bar{k}_{p}\right)_{p}^{+}=0$, which exhibits $\widetilde{Q}_{p}$ as the operator governing the 26 limit problems for any triplet $p \in\{0,1,2\}^{3} \backslash$ $\{0,0,0\}$.

The limit space of phonon fields will be the space of Kirchhoff-Love displacements defined by $V_{K L}=\left\{v \in H_{\Gamma_{u D}}^{1}(\Omega)^{3}\right.$; $\left.e_{i 3}(v)=0\right\}$ while the limit phonon fields need the introduction of nine spaces $\Psi_{0, i}=\{0\}, \Psi_{1, i}=\left\{\phi \in H_{\Gamma_{w D, i}}^{1}(\Omega) ; \partial_{3} \phi=0\right\}$ and $\Psi_{2, i}=\left\{\phi \in H_{\partial_{3}}^{1}(\Omega) ; \phi=0\right.$ on $\left.\Gamma_{w D, i} \cap \Gamma^{ \pm}\right\}$where $H_{\partial_{3}}^{1}(\Omega)=\left\{\phi \in L^{2}(\Omega) ; \partial_{3} \phi \in L^{2}(\Omega)\right\}$. The space of limit physical states is defined as $\mathbf{V}_{p}=V_{K L} \times \Psi_{p_{1}, 1} \times \Psi_{p_{2}, 2} \times \Psi_{p_{3}, 3}$. Under assumptions $\left(\mathbf{H}_{1}\right)-\left(\mathbf{H}_{5}\right)$, we have the following convergence result:

Theorem 3.1. Let $p \in\{1,2,3\}^{3} \backslash\{0,0,0\}, X_{0}=X_{1}=H^{1}(\Omega)$ and $X_{2}=H_{\partial_{3}}^{1}(\Omega)$. When $\varepsilon$ tends to 0 , the family $\left(s_{p}(\varepsilon)\right)_{\varepsilon>0}$ of the unique solutions of $\mathcal{P}(\varepsilon, \Omega)_{p}$ strongly converges in $\mathbf{X}_{p}=H_{\Gamma_{u D}}^{1}(\Omega)^{3} \times X_{p_{1}} \times X_{p_{2}} \times X_{p_{3}}$ to the unique solution $\bar{s}_{p}$ of:

$$
\overline{\mathcal{P}}(\Omega)_{p}: \quad \text { Find } \bar{s}_{p} \in\left(0, w_{0}\right)+\mathbf{V}_{p} \quad \text { such that } \int_{\Omega} \widetilde{\mathrm{Q}}_{p}(x) k\left(\bar{s}_{p}\right)_{p}^{0} \cdot k(\xi)_{p}^{0} \mathrm{~d} x=L(\xi), \quad \forall \xi \in \mathbf{V}_{p}
$$

where $k(\xi)$ stands for $(e(v), \nabla \psi)$, while $k(\xi)_{p}^{0}$ denotes the projection of $k(\xi)$ on the space $\mathbb{H}_{p}^{0}$, for all $\xi=(v, \psi) \in \mathbf{V}_{p}$.

Proof. Actually, the problem can be considered as a "vectorial variant" of the asymptotic behavior of thin piezoelectric plates [6] where each component of the phason plays the role of an electrical potential. Hence all the arguments of [6] can be used; the additional case $p_{i}=0$ being obviously handled through the assumptions $\left(\mathbf{H}_{1}\right)-\left(\mathbf{H}_{5}\right)$ which, together with Poincaré inequality, imply the boundedness of $\left|\left(\nabla_{p}\left(\varepsilon, w_{p}(\varepsilon)\right)\right)_{i j}\right|_{L^{2}(\Omega)}, 1 \leqslant j \leqslant 3$. The case $p=(0,0,0)$ is not considered here because it leads to a Kirchhoff-Love plate model without contribution of phason fields.

To get physically meaningful results it is necessary to define a physical state $\bar{s}_{p}^{\varepsilon}$ over the genuine plate $\Omega^{\varepsilon}$ through the descaling $\bar{s}_{p}^{\varepsilon}\left(\pi^{\varepsilon} x\right)=\bar{s}_{p}(x)$, for all $x$ in $\Omega$. This physical state is the unique solution of a problem posed over the real plate, namely:

$$
\overline{\mathcal{P}}\left(\Omega^{\varepsilon}\right)_{p}: \quad \text { Find } \bar{s}_{p}^{\varepsilon} \in\left(0, w_{0}^{\varepsilon}\right)+\mathbf{V}_{p}^{\varepsilon} \quad \text { such that } \int_{\Omega^{\varepsilon}} \widetilde{Q}_{p}^{\varepsilon}(x) k\left(\bar{s}_{p}^{\varepsilon}\right)_{p}^{0} \cdot k(\xi)_{p}^{0} \mathrm{~d} x=L^{\varepsilon}(\xi), \quad \forall \xi \in \mathbf{V}_{p}^{\varepsilon}
$$

The superscript $\varepsilon$ in $\mathbf{V}_{p}^{\varepsilon}$ and $L^{\varepsilon}$ means that $\Omega$ is replaced by $\Omega^{\varepsilon}$ in the definition of $\mathbf{V}_{p}$ and $L$, respectively. This transported problem is our proposal to model thin linearly quasicrystalline plates of thickness $2 \varepsilon$. In these models, constitutive laws are given by $\widetilde{Q}_{p}^{\varepsilon}$ such that $\widetilde{Q}_{p}^{\varepsilon}\left(\pi^{\varepsilon} x\right)=\widetilde{Q}_{p}(x)$, for all $x$ in $\Omega$. We enlighten some remarkable properties of these constitutive laws in the next section.

\section{Some properties of $\widetilde{Q_{p}^{\varepsilon}}$ for icosahedral quasicrystals}

The convergence result stated in Theorem 3.1 is of course valid for any quasicrystalline class. However, in all about 200 individual quasicrystals observed to date, a wide majority presents an icosahedral order. This suggests that this symmetry plays a central role in the study of the physical behavior of quasicrystalline media. For such symmetry, there are two elastic coefficients $\lambda^{\varepsilon}$ and $\mu^{\varepsilon}$ in tensor $C^{\varepsilon}$, one coupling coefficient $r^{\varepsilon}$ in tensor $R^{\varepsilon}$ and two coefficients $K_{1}^{\varepsilon}$ and $K_{2}^{\varepsilon}$ in tensor $K^{\varepsilon}$ (see relation (3)). We now focus our attention on the limit operators $\widetilde{Q}_{p}^{\varepsilon}$ which supply the constitutive relations of the quasicrystalline plate. These limit operators can be computed directly from the explicit expression (10). In spite of the dimension reduction process, it is possible to show that they keep the same structure than $Q^{\varepsilon}($ see $(2))$ in the sense that

$$
\widetilde{Q}_{p}^{\varepsilon}=\left(\begin{array}{cc}
\widetilde{C}_{p}^{\varepsilon} & \widetilde{R}_{p}^{\varepsilon} \\
\widetilde{R}_{p}^{\varepsilon^{T}} & \widetilde{K}_{p}^{\varepsilon}
\end{array}\right)
$$

where $\widetilde{C}_{p}^{\varepsilon}, \widetilde{R}_{p}^{\varepsilon}$ and $\widetilde{K}_{p}^{\varepsilon}$ respectively denote the limit tensors of phonon, phonon-phason coupling and phason coefficients. These coefficients involve a mixture of the five coefficients $\lambda^{\varepsilon}, \mu^{\varepsilon}, r, K_{1}^{\varepsilon}$ and $K_{2}^{\varepsilon}$. More precisely, it is possible to show that we have the following properties:

- $\widetilde{C}_{p}^{\varepsilon}$ always involves a mixture of the later five physical coefficients, except for $p=(2,2,2)$ for which $K_{2}^{\varepsilon}$ is not present,

- for $p \in\{(1,1,2),(1,2,2),(2,1,2),(2,2,2),(2,0,2),(0,1,2),(0,2,2)\}, \widetilde{K}_{p}^{\varepsilon}$ involves all the five physical coefficients,

- for $p \in\{(1,1,1),(1,1,0),(1,2,1),(1,2,0),(1,0,1),(1,0,2),(1,0,0),(2,1,1),(2,1,0),(2,2,1),(2,0,1),(0,1,1)$, $(0,1,0),(0,2,1),(0,0,1),(0,0,2)\}, \widetilde{K}_{p}^{\varepsilon}$ involves $\mu^{\varepsilon}, r^{\varepsilon}, K_{1}^{\varepsilon}$ and $K_{2}^{\varepsilon}$ but not $\lambda^{\varepsilon}$, 
- for $p \in\{(2,2,0),(2,0,0),(0,2,0)\}, \widetilde{K}_{p}^{\varepsilon}$ involves only $K_{1}^{\varepsilon}$ and $K_{2}^{\varepsilon}$,

- for $p \in\{(1,1,2),(1,2,2),(1,2,0),(1,0,2),(2,1,2),(2,2,2),(2,0,2),(0,1,2),(0,2,2)\}, \widetilde{R}_{p}^{\varepsilon}$ involves all the five physical coefficients,

- for $p \in\{(1,2,1),(1,0,1),(1,0,0),(2,1,1),(2,1,0),(0,1,1),(0,1,0)\}, \widetilde{R}_{p}^{\varepsilon}$ involves $\mu^{\varepsilon}, r^{\varepsilon}, K_{1}^{\varepsilon}$ and $K_{2}^{\varepsilon}$ but not $\lambda^{\varepsilon}$,

- for $p \in\{(1,1,1),(1,1,0),(2,2,1),(2,2,0),(2,0,1),(2,0,0),(0,2,1),(0,2,0)\}, \widetilde{R}_{p}^{\varepsilon}$ involves $r^{\varepsilon}, K_{1}^{\varepsilon}$ and $K_{2}^{\varepsilon}$ but not $\lambda^{\varepsilon}$ neither $\mu^{\varepsilon}$,

- for $p \in\{(0,0,1),(0,0,2)\}, \widetilde{R}_{p}^{\varepsilon}$ vanishes: for quasicrystalline plates submitted to such type and magnitude of boundary conditions, the physical coupling between phonons and phasons disappears at the structural level! Moreover, the tensor $\widetilde{K}_{p}^{\varepsilon}$ is diagonal.

As an example, for $p=(2,2,2)$ we have

$$
\begin{aligned}
& \widetilde{C}_{(2,2,2)}^{\varepsilon}=\left(\begin{array}{cccc}
\frac{2\left(2 \mu^{\varepsilon} K_{1}^{\varepsilon}-r^{\varepsilon^{2}}\right)\left(\lambda^{\varepsilon}+2 \mu^{\varepsilon}\right)}{\left(\lambda^{\varepsilon}+2 \mu^{\varepsilon}\right) K_{1}^{\varepsilon}} & \frac{2 \mu^{\varepsilon} \lambda^{\varepsilon} K_{1}^{\varepsilon}+2 r^{\varepsilon^{2}}\left(\lambda^{\varepsilon}+2 \mu^{\varepsilon}\right)}{\left(\lambda^{\varepsilon}+2 \mu^{\varepsilon}\right) K_{1}^{\varepsilon}} & 0 & 0 \\
\frac{2 \mu^{\varepsilon} \lambda^{\varepsilon} K_{1}^{\varepsilon}+2 r^{\varepsilon^{2}}\left(\lambda^{\varepsilon}+2 \mu^{\varepsilon}\right)}{\left(\lambda^{\varepsilon}+2 \mu^{\varepsilon}\right) K_{1}^{\varepsilon}} & \frac{2\left(2 \mu^{\varepsilon} K_{1}^{\varepsilon}-r^{\varepsilon^{2}}\right)\left(\lambda^{\varepsilon}+2 \mu^{\varepsilon}\right)}{\left(\lambda^{\varepsilon}+2 \mu^{\varepsilon}\right) K_{1}^{\varepsilon}} & 0 & 0 \\
0 & 0 & 2 \frac{\mu^{\varepsilon} K_{1}^{\varepsilon}-r^{\varepsilon^{2}}}{K_{1}^{\varepsilon}} & -2 \frac{r^{\varepsilon^{2}}}{K_{1}^{\varepsilon}} \\
0 & -2 \frac{r^{\varepsilon^{2}}}{K_{1}^{\varepsilon}} & 2 \frac{\mu^{\varepsilon} K_{1}^{\varepsilon}-r^{\varepsilon^{2}}}{K_{1}^{\varepsilon}}
\end{array}\right) \\
& \widetilde{R}_{(2,2,2)}^{\varepsilon}=\left(\begin{array}{ccc}
\frac{\left(K_{1}^{\varepsilon}-2 K_{2}^{\varepsilon}\right) r^{\varepsilon}}{K_{1}^{\varepsilon}} & 0 & \frac{r^{\varepsilon}\left(3 \lambda^{\varepsilon}+2 \mu^{\varepsilon}\right)}{\lambda^{\varepsilon}+2 \mu^{\varepsilon}} \\
-\frac{\left(K_{1}^{\varepsilon}-2 K_{2}^{\varepsilon}\right) r^{\varepsilon}}{K_{1}^{\varepsilon}} & 0 & \frac{r^{\varepsilon}\left(3 \lambda^{\varepsilon}+2 \mu^{\varepsilon}\right)}{\lambda^{\varepsilon}+2 \mu^{\varepsilon}} \\
0 & -\frac{\left(K_{1}^{\varepsilon}-2 K_{2}^{\varepsilon}\right) r^{\varepsilon}}{K_{1}^{\varepsilon}} & 0 \\
0 & -\frac{\left(K_{1}^{\varepsilon}-2 K_{2}^{\varepsilon}\right) r^{\varepsilon}}{K_{1}^{\varepsilon}} & 0
\end{array}\right) \\
& \widetilde{K}_{(2,2,2)}^{\varepsilon}=\left(\begin{array}{ccc}
\frac{K_{1}^{\varepsilon^{2}}-K_{2}^{\varepsilon} K_{1}^{\varepsilon}-2 K_{2}^{\varepsilon^{2}}}{K_{1}^{\varepsilon}} & 0 \\
0 & \frac{K_{1}^{\varepsilon^{2}-}-K_{2}^{\varepsilon} K_{1}^{\varepsilon}-2 K_{2}^{\varepsilon^{2}}}{K_{1}} & 0 \\
0 & 0 & \frac{\left(\lambda^{\varepsilon}+2 \mu^{\varepsilon}\right)\left(K_{1}^{\varepsilon}+K_{2}^{\varepsilon}\right)-4 r^{\varepsilon^{2}}}{\lambda^{\varepsilon}+2 \mu^{\varepsilon}}
\end{array}\right)
\end{aligned}
$$

\section{References}

[1] D. Shechtman, I. Blech, D. Gratias, J.W. Cahn, Metallic phase with long-range orientational order and no translational symmetry, Phys. Rev. Lett. 53 (1984) 1951-1953.

[2] P.J. Steinhardt, S. Ostlund (Eds.), The Physics of Quasicrystals, World Scientific, Singapore, 1987.

[3] A. Blaaderen, Quasicrystals from nanocrystals, Mater. Sci. 461 (2009) 892-893.

[4] J.-M. Dubois, Useful Quasicrystals, World Scientific, London, 2005.

[5] J.-M. Dubois, New prospects from potential applications of quasicrystalline materials, Mater. Sci. Eng. 294-296 (2000) 4-9.

[6] T. Weller, C. Licht, Asymptotic modeling of thin piezoelectric plates, Ann. Solid Struct. Mech. 1 (2010) 173-188.

[7] T. Weller, C. Licht, Mathematical modeling of piezomagnetoelectric thin plates, Eur. J. Mech. A, Solids 29 (2010) 928-937.

[8] T. Weller, C. Licht, Asymptotic modeling of piezoelectric plates with electric field gradient, C. R., Mecanique 340 (2012) 405-410.

[9] T. Weller, C. Licht, Asymptotic modeling of linearly piezoelectric slender rods, C. R., Mecanique 336 (2008) 572-577.

[10] D.H. Ding, W.G. Yang, C.Z. Hu, R. Wang, Generalized elasticity theory of quasicrystals, Phys. Rev. B 48 (1993) 7003-7010.

[11] T. Fan, Mathematical Theory of Elasticity of Quasicrystals and Its Applications, Springer, 2011.

[12] D. Levine, T.C. Lubensky, S. Ostlund, S. Ramaswamy, P.J. Steinhardt, Elasticity and dislocations in pentagonal and icosahedral quasicrystals, Phys. Rev. Lett. 54 (1985) 1520-1523.

[13] H. Brézis, Functional Analysis, Sobolev Spaces and Partial Differential Equations, Springer, 2011.

[14] P.G. Ciarlet, Mathematical Elasticity, vol. II, North-Holland, 1997. 